\title{
Radiation Health Risk Communication in Nagasaki University/Kawauchi Village Reconstruction Promotion Base
}

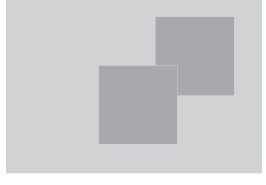

\author{
Nagasaki University, Makiko Orita
}

In March 2011, an accident occurred at the Fukushima Daiichi Nuclear Power Plant, which is operated by the Tokyo Electric Power Company, due to the Tohoku earthquake and ensuing tsunami (such accident being hereinafter referred to as the "Fukushima Daiichi Nuclear Accident"). The resultant environmental release of invisible, scentless radioactive materials, which are undetectable by human senses, caused a serious panic among the public. The incident created public interest in radiation health risk communication, which addresses the health impact of exposure to radiation. According to conventional wisdom, radiation health risk communication is pursued among experts, government officials, and community members with the aim of sharing information on the health risks posed by radiation and making mutual communication among them. Today, radiation health risk communication tailored to the living conditions and ideas of individuals according to the air dose rates and exposure dose rates seems to be of increasing importance.

On April 20, 2013, Kawauchi Village in Futaba, Fukushima Prefecture, signed an agreement with Nagasaki University on comprehensive cooperation. Since then, the author has engaged in various healthcare activities at the reconstruction promotion base of Nagasaki University that they established in Kawauchi Village to protect community members from the health risks associated with radiation. This commentary summarizes these activities.

\section{Kawauchi Village in Futaba, Fukushima Prefecture}

Kawauchi, a village located in Futaba, Fukushima Prefecture had a total population of about 3,000 when the disaster hit the area. This scenic part of the countryside is nestled in the hills alongside the Abukuma Mountains in the prefecture's Hamadori region. It lies within 20 to $30 \mathrm{~km}$ of the Fukushima Daiichi Nuclear Power Plant. Affected by the Fukushima Daiichi Nuclear Accident that occurred in March 2011, all of the village members, along with the village office personnel, evacuated to Koriyama (which is located in the same prefecture). In January 2012, Kawauchi Village declared before any other village its intention to allow its citizens to return home in light of the relatively low air dose rates there. The village office

DOI : 10.15669/fukushimainsights.Vol.3.133

(C) 2021 Atomic Energy Society of Japan. All rights reserved.

Originally published in Journal of the Atomic Energy Society of Japan (ISSN 1882-2606), Vol. 57, No. 5, p. 346-349 (2015)

in Japanese. (Japanese version accepted: February 19, 2015) 
moved its administrative functions back to Kawauchi from Koriyama at the end of March 2012 while encouraging community members to return home whenever they were ready. Efforts have been steadily made to plan the decontamination work, restore farming and forestry, and stimulate local commerce with the aim of rebuilding the community.

As of January 2015, about 1,600 residents of the total population of roughly 2,700 have returned to Kawauchi. A gradual return has continued since the official declaration was made. However, about $40 \%$ of the villagers continue to live in other municipalities as evacuees. The possible causes of their reluctance to return home almost certainly include concerns over the village's educational and healthcare infrastructure as well as convenience in their daily lives. However, they might also have been discouraged by risks associated with the decommissioning work being carried out at the Fukushima Daiichi Nuclear Power Plant and concerns over the health effects caused by exposure to radiation.

\section{Kawauchi Village Reconstruction Promotion Base of Nagasaki University}

Since December 2011, Nagasaki University has been assisting in the reconstruction efforts carried out by Kawauchi Village. For instance, the university estimated the exposure dose rates among residents to establish scientific grounds for their intended return by measuring the radioactive materials contained in the soil in December 2011. The university communicated these findings to village residents at lecture meetings and the like. It also provided scientific support for efforts by village members to return home and restore their community.

On April 20, 2013, Nagasaki University and Kawauchi Village signed an agreement on comprehensive cooperation for the restoration and revival of the local community. In the village, the Kawauchi Village Reconstruction Promotion Base was established as a satellite facility of Nagasaki University. This base works closely with the village office to measure radioactivity in the soil and food to ensure the safety of residents as well as offer radiation health consultation services based on the measurement data. At the base, local health nurses work with experts in community-based rehabilitation and community heath from Nagasaki University's School of Medicine (health nurses, physiotherapists, and occupational therapists). They organize various health counseling sessions, exercise therapy, and other healthpromoting activities in combination with social gatherings and other events for community members. Notably, the base brings together experts (from Nagasaki University) and the local government (village office of Kawauchi) to promote local reconstruction. This expertgovernment partnership is expected to facilitate effective radiation health risk communication.

\section{Activities Involving Radiation Health Risk Communication}

At the Kawauchi Village Reconstruction Promotion Base, the author has engaged in healthcare activities to protect community members from the health risks associated with radiation. This engagement began when the author stayed in the village for a month from May 2012 immediately after the evacuees had begun to return. As part of the field practice element of her master's program, she worked with local health nurses to provide health consultations on radiation and perform door-to-door visits. When the village and Nagasaki University 
signed the cooperation agreement in April 2013, she was permanently assigned to the satellite base to continue her work there. Local health nurses conduct a wide range of healthcare activities, including maternal and child healthcare, geriatric healthcare, mental healthcare, community health promotion, and infection control. The satellite base mainly took charge of healthcare activities for the provision of protection against radiation. Located in the only city other than Hiroshima to have been attacked with an atomic bomb, Nagasaki University has been studying the health impact of radiation exposure and the carcinogenic mechanism of radiation. It has also played a role in the provision of healthcare to atomic bomb survivors. The university is expected to apply their findings and engage in tangible activities in response to the Fukushima Daiichi Nuclear Accident. The satellite base, which is situated in the community center (locally known as Nakayoshi-kan) near the village office (Figure 1), is gradually gaining recognition among residents thanks to the public relations activities conducted through the village office and the announcements made at the social gatherings that are frequently organized in the village and at shelters for evacuees from the village.

In most cases, teams from the base conduct door-to-door visits to provide consultation services. Residents often ask questions such as whether the water and rice are safe for consumption, whether children can touch insects, and whether any decontamination work was carried out after the atomic bomb was dropped on Nagasaki. The teams also attend social gatherings organized by the village to support evacuees, discuss future land use, and other such purposes (Figure 2). At these gatherings, the teams respond to questions from residents related to radiation and health as well as distribute dosimeters that have been donated for the residents along with the instructions for their proper use (Figure 3).

During consultations on health and radiation, people often ask how they should interpret the various figures. The nuclear accident was followed by panic and a flood of information and unfamiliar terms, such as $\mu \mathrm{Sv}$, Becquerel, and internal exposure. As time went by, air dose detectors were deployed all over Fukushima Prefecture. Furthermore, local dose rates are reported daily in detail by newspapers. In fact, dosimeters are so widely used that almost every villager from Kawauchi has at least one personal dosimeter, which means that they can find out their own radiation doses. Nonetheless, measures aimed at helping them to interpret these doses are falling behind. Given this situation, Nagasaki University has prioritized healthcare activities aimed at helping people learn how to interpret these figures.

The conducting of dose assessments is an important starting part for radiation health risk

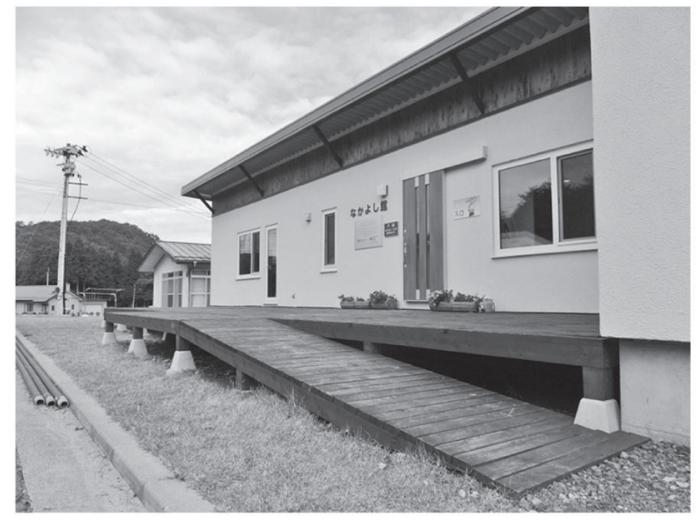

Figure 1 A community center where the satellite base of Nagasaki University is situated. (Kawauchi, October 2013) 


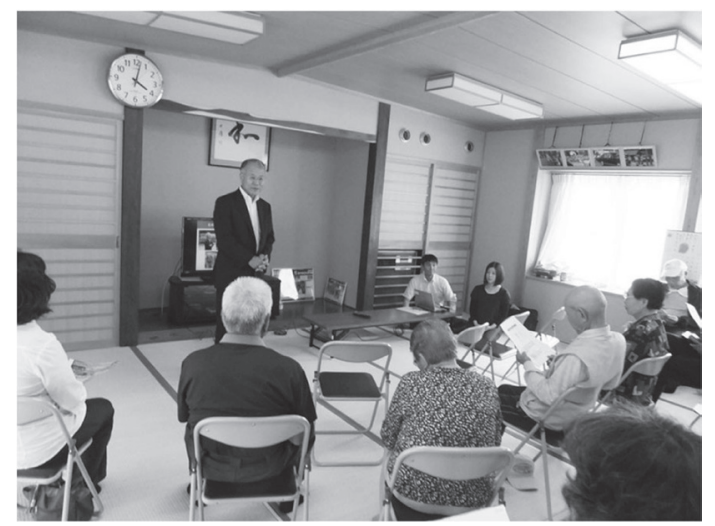

Figure 2 A briefing session for local residents at a community hall (Kawauchi, June 2014)

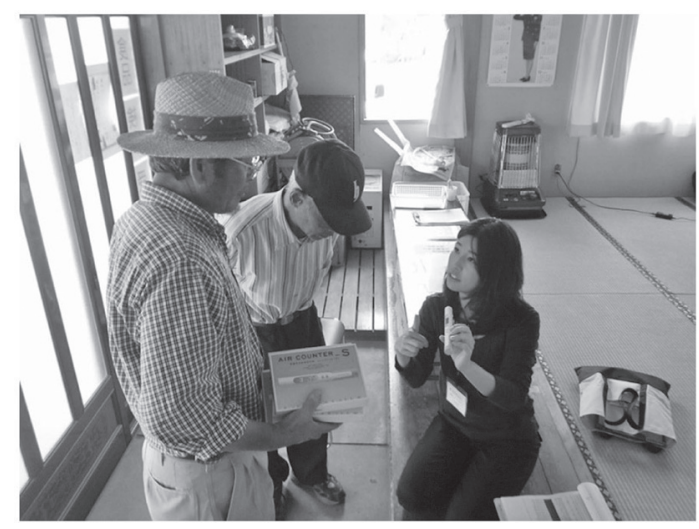

Figure 3 Distribution of dosimeters at a community hall (Kawauchi, June 2013)

communication. The base teams conduct measurements of the air dose rates at the homes of villagers and in the surrounding areas. They also bring back soil and vegetable samples for radioactivity measurements. The teams present these measurements to villagers and explain what each of the figures indicates. In this manner, the teams address the villagers' questions and concerns over radiation exposure in everyday life. The villagers often comment that they feel reassured if they can understand the levels of radioactivity in local soil and food.

In May 2014, a germanium detector was installed and put into service in Kawauchi Village thanks to the cooperation of the Nuclear Safety Research Association. So far, Kawauchi Village has deployed a simplified detector in each district and conducted radioactivity tests on food. At the request of the villagers, Nagasaki University also conducted radioactivity measurements back in Nagasaki by sending the food entrusted to them there. In addition to the ongoing food tests, the germanium detector has also made it possible for much-needed measurements on soil and water to be performed quickly. Health consultations and other forms of radiation health risk communication should probably be based on objective numerical assessments of the radiation. 


\section{Efforts to Facilitate a Return to the Evacuation-Directive Area in Kawauchi Village}

Kawauchi Village had been zoned as a "restricted area" and an "evacuation-prepared area in case of emergency" since April 2011. The latter designation was lifted in fall 2011. The restricted area within $20 \mathrm{~km}$ of the Fukushima Daiichi Nuclear Power Plant was rezoned as an "area in which residents are not permitted to live" and an "area to which evacuation orders are ready to be lifted" in April 2012. In both cases, residents have been permitted to freely access their homes, but not stay there overnight. Meanwhile, due to encouraging signs that the decontamination work is near completion, efforts have been made to support the return of evacuees to these areas in Kawauchi.

The various studies conducted after the nuclear accident revealed that the measured personal doses do not necessarily match the exposure doses estimated from the air dose rates based on the assumption that residents will exhibit the same behavior and that homes will have the same shield factor ${ }^{1)}$. For this reason, Fukushima is expected to facilitate the return of willing evacuees by implementing the necessary measures while paying attention to their individual exposure doses. To this end, Nagasaki University has been assessing the environmental radioactivity and individual exposure doses in the evacuation-directive area. More specifically, the university has distributed personal dosimeters to residents with special permission to stay in their homes overnight during occasions such as the New Year holidays. Their individual doses have been assessed to estimate their annual exposure doses. In combination with an assessment of the air doses and analysis of the radionuclides in soil, the validity of a return to the evacuation-directive area has been evaluated.

To date, personal dosimeters have registered, in annual terms, a minimum annual dose of $0.71 \mathrm{mSv}$, a maximum dose of $2.15 \mathrm{mSv}$, an average dose of $1.25 \mathrm{mSv}$, and a median dose of $1.21 \mathrm{mSv}$. The annual average dose slightly exceeds the annual exposure dose limit of $1 \mathrm{mSv}$ for the public under ordinary circumstances, as set by the International Commission on Radiological Protection (ICRP). However, such a dose proved extremely limited. Meanwhile, air dose rates were measured when villagers were given special permission to stay home overnight when they returned home temporarily. The total annual dose in each house was calculated based on measurements taken outside the front doors of houses, behind the houses, and in fields. In annual terms, the measurements taken outside the front doors registered a minimum dose of $0.88 \mathrm{mSv}$, a maximum dose of $1.75 \mathrm{mSv}$, an average dose of $1.14 \mathrm{mSv}$, and a median dose of $1.04 \mathrm{mSv}$. These figures were almost comparable to or slightly lower than the measured individual exposure doses. This is probably due to the successful dose reduction achieved by the almost complete decontamination of houses in areas to which evacuation orders are ready to be lifted. Similarly, the measurements taken behind the houses registered a minimum dose of $0.99 \mathrm{mSv}$, a maximum dose of $2.15 \mathrm{mSv}$, an average dose of $1.63 \mathrm{mSv}$, and a median dose of $1.58 \mathrm{mSv}$. The measurements taken in fields registered, in annual terms, a minimum dose of $1.45 \mathrm{mSv}$, a maximum dose of $3.29 \mathrm{mSv}$, an average dose of $1.82 \mathrm{mSv}$, and a median dose of $1.77 \mathrm{mSv}$. As expected, the doses recorded in fields were slightly higher than the ones recorded around the houses. Based on these measurement results, the annual external exposure doses are expected to be within the range of 1 to $2 \mathrm{mSv}$ at most, which validates the return of evacuees to their homes. The massive epidemiological studies that have been conducted so far on atomic bomb survivors from Hiroshima and Nagasaki revealed that survivors experienced an increase in cancer risk in proportion to any exposure doses in excess of $100 \mathrm{mSv}$ at one time. However, no increase in cancer risk has been proven with an exposure dose of below $100 \mathrm{mSv}$. The ICRP has set a limit of $1 \mathrm{mSv}$ as an acceptable annual 
exposure dose for the public under ordinary circumstances to minimize any additional exposure to radiation. It must be noted, however, that the cancer risk resulting from such exposure does not begin to increase from this threshold of $1 \mathrm{mSv}$. Moreover, during a radiation disaster, the ICRP recommends that the limit for the annual exposure dose be assigned to the lowest possible level of between 20 to $100 \mathrm{mSv}$. Once the disaster has been brought under control, the ICRP recommends that the annual limit be gradually reduced before it ultimately reaches $1 \mathrm{mSv}$. Given the outcomes of earlier decontamination work and other efforts to reduce exposure doses in Kawauchi, the village office deemed it appropriate for evacuees to return home as long as the effects of decontamination are continuously evaluated, infrastructure is steadily improved, and the community is rebuilt under the ongoing partnership among residents, the village office, and experts from various fields. Accordingly, the designation of "the area to which evacuation orders are ready to be lifted" was lifted on October 1, 2014. Nonetheless, the monitoring of external exposure doses should be continued in response to any later requests from villagers along with the provision of fine-tuned consultation services according to exposure doses.

The establishment of a satellite base by Nagasaki University in Kawauchi Village to act as a frontline for post-disaster reconstruction efforts made it possible to keep track of the needs of local residents and any questions that they encountered, thereby facilitating radiation health risk communication. Furthermore, a backup system for the university staff that permanently stays at the village was gradually brought online to enable resident personnel to consult with experts in Nagasaki University and address any issues and/or questions that they may encounter during the performance of local activities while identifying the needs of the villagers. Almost five years after the disaster, many more evacuees are expected to return to the municipalities situated around the Fukushima Daiichi Nuclear Power Plant. Assistance for these returning evacuees must be carefully planned. The national government is planning to assign community counselors on radiation exposure and health. Their assignments will probably need to be clarified, and the necessary backup system should be established for them. In light of the need for long-term assistance, urgent human resource development in the field of radiation exposure medicine and healthcare professionals is needed to ensure that they are adept in radiation emergency medicine. The healthcare system in Fukushima is clearly being dutifully underpinned by the Fukushima Medical University in Fukushima City. This university is expected to lead the process of building up the more necessary systems. At the same time, a collaborative system should be established between counselors and experts as a part of a bigger framework for supporting the university.

\section{Challenges Ahead}

In radiation health risk communication, it is important to respect the views that each of the local residents has concerning exposure to radiation and their ultimate decisions as to whether to return home or remain as evacuees. Healthcare professionals and experts must help local residents to make informed decisions.

Meanwhile, attention has been drawn to the dire shortage of experts who can engage in radiation health risk communication. One of the challenges that lie ahead is how these experts should be developed. Public health and other nurses are known to be familiar with the actual living conditions in the local communities there, so they play extremely important roles during a nuclear disaster and in every step of the reconstruction process. Unfortunately, these 
nurses had not received much education in radiological protection, let alone radiation emergency medicine. As an educational institution situated in a city that has suffered from an atomic bomb attack, Nagasaki University has been conducting lectures on radiation exposure and nursing for almost a decade as part of an undergraduate program in its School of Health Sciences. These lectures are intended to help students gain a deeper understanding of their expected roles in radiation emergency medicine as public health and other nurses in the future. This type of undergraduate program is offered in only a few universities. In 2010, Nagasaki University began offering a master's course for the development of public health and other nurses specializing in emergency radiation medicine. The author has also attended this course to acquire a basic understanding of radiological protection and radiological impacts as well as to study the roles that public health and other nurses are expected to fulfill in the provision of radiation emergency medicine. Having completed the master's program there, she is now enrolled in a doctoral program at the Atomic Bomb Disease Institute in the same university. At present, only three universities in Japan-Hirosaki University, Nagasaki University, and Kagoshima University — offer master's courses in radiation nursing. The annual course enrollment numbers account for only a few students in each graduate school. In light of the long-term continuous efforts that are required for reconstruction after a nuclear accident, it is becoming ever more important to involve public health and other nurses together with other healthcare professionals who are familiar with radiation emergency medicine. As evacuees from Fukushima Prefecture municipalities situated within $20 \mathrm{~km}$ of the disaster site continue to return to their homes, such personnel all play essential roles. Having acquired the minimum level of expertise required in radiological protection and radiation emergency medicine, public health and other nurses are not only able to provide better healthcare under ordinary circumstances, but also well placed to help plan how experts are mobilized in nuclear disaster management.

\section{Conclusions}

In the practice of radiation health risk communication, local government agencies and experts should work together to attentively support local residents. Furthermore, with radiation health risk communication in post-disaster reconstruction continuing to attract increasing attention, the relevant activities conducted in Kawauchi Village could serve as a model for further reconstruction efforts throughout the rest of Fukushima.

\section{Reference}

1) Nuclear Regulation Authority. Practical Measurements for Evacuees to Return to Their Homes. http:// www.nsr.go.jp/english/Library/data/specialreport_20140204.pdf. 